\title{
Infrared imaging simulation through anisotropic atmosphere turbulence
}

\author{
Dongjie Tan ${ }^{1, a}$ \\ ${ }^{1}$ School of Aeronautics Science and Engineering, Beihang University, Beijing 100191,China \\ atandongjie2015@sina.com
}

\begin{abstract}
Keywords: turbulence simulation, anisotropic turbulence, angle of arrival fluctuations, modulation transfer function.
\end{abstract}

\begin{abstract}
Atmosphere turbulence often degrades image quality in long range infrared imaging system due to intensity fluctuations, distortion, and blur effects. In this work, long range infrared imaging through anisotropic turbulence is simulated, which combines the theoretical turbulence effects models of optical waves (the angle of arrival fluctuations of optical wave and the modulation transfer function) under anisotropic non-Kolmogorov turbulence and the imaging processing algorithms (image convolution and interpolation methods). This simulation technique allows the performance assessment of a long range infrared imaging system under various anisotropic atmosphere turbulence conditions to be done in a cost efficient manner. Simulation uses single image containing no turbulence effects as an input and produces image sequences degraded by specified turbulence. Imaging distance, optics diameter, wavelength, anisotropic factor, general spectral power law, and turbulence strength are included. The influence of anisotropic non-Kolmogorov turbulence on the long range infrared imaging system is analyzed.
\end{abstract}

\section{Introduction}

Imaging through atmosphere turbulence is an inevitable problem encountered by infrared imaging sensors working in atmosphere turbulence environment. Turbulence causes the light to arrive at imaging sensor at slightly different angles, which can introduce intensity fluctuations, distortion, and blur effects to the imaging system. Large field campaigns to measure imaging performance in the presence of turbulence are often cost prohibitive. In comparison, the computer simulation is a more cost efficient way, which can consistently add turbulence to a pristine target set and helps assess the imaging system performance or evaluate turbulence mitigation algorithms under various turbulence conditions.

Conventional numerical simulation of imaging through atmosphere turbulence is typically performed with the well-known split-operator method [1-4], which adopts a set of thin phase screen to describe the phase variations introduced by the atmosphere turbulence. This method produces high accuracy simulations, but it is computationally intensive and very time consuming. The image-based turbulence imaging simulations run quickly by adopting image processing methods such as image resampling and filtering [5-8]. The image blur and distort produced by atmosphere turbulence can be obtained with the theoretical models of atmosphere turbulence effects. The simulation uses a single image with no turbulence effects as input and produces degraded sequences containing temporal and spatial turbulence effects. These simulations have been mainly focused on the isotropic turbulence for years. The sizes of turbulence cells or eddies in the horizontal and vertical directions are the same.

However, experiments and theoretical investigations have shown that the atmosphere turbulence also exhibits anisotropic and non-Kolmogorov properties [9-15]. Compared with isotropic turbulence, asymmetry properties of turbulence cells or eddies in horizontal and vertical directions are exhibited. In the investigations, atmosphere turbulence refractive-index fluctuations spectrum which describes the statistical property of the random fluctuations of atmosphere refractive-index is the most basic model. The anisotropic non-Kolmogorov turbulence spectral model was proposed in [11], and the anisotropic factor was introduced to parameterize the asymmetry of turbulence cells or eddies in horizontal and vertical directions. It has been widely applied in the theoretical investigations of optical waves propagating through anisotropic non-Kolmogorov turbulence $[11,14,15]$. 
In this work, we focus on the long-range infrared imaging simulation in anisotropic non-Kolmogorov turbulence. Based on the anisotropic non-Kolmogorov turbulence effects models established with the spectral model reported in [11] and the image processing algorithms (the image convolution and the interpolation methods), images degraded by anisotropic non-Kolmogorov turbulence will be simulated. In the simulations, the anisotropic factor which parameterizes the asymmetry of turbulence cells or eddies in the horizontal and vertical directions, the general spectral power law in the range of 3-4 instead of standard value of 11/3 for the Kolmogorov turbulence, the turbulence refractive-index structure constant, the imaging distance, the receiver aperture, and the wavelength will be considered synthetically.

\section{Anisotropic non-Kolmogorov turbulence refractive-index fluctuations spectrum}

The anisotropic non-Kolmogorov power spectrum over the inertial sub-range takes the form as [11]:

$$
\begin{gathered}
\Phi_{n}(\kappa, \alpha, \varsigma)=A(\alpha) \cdot \hat{C}_{n}^{2} \cdot \varsigma^{2} \cdot\left[\kappa_{z}^{2}+\varsigma^{2}\left(\kappa_{x}^{2}+\kappa_{y}^{2}\right)\right]^{-\frac{\alpha}{2}} \cdot\left(1 / L_{0}<\kappa<1 / l_{0}, 3<\alpha<4\right), \\
A(\alpha)=\frac{1}{4 \pi^{2}} \Gamma(\alpha-1) \cos \left[\frac{\alpha \pi}{2}\right] .
\end{gathered}
$$

where $\alpha$ is the general spectral power law value, $\hat{C}_{n}^{2}$ is the generalized structure parameter with units $m^{3-\alpha}$, and $\Gamma(\cdot)$ is the gamma function. $\kappa$ is the wavenumber related to the turbulence cell size, $\kappa=\sqrt{\kappa_{x}^{2}+\kappa_{y}^{2}+\kappa_{z}^{2}} \cdot \kappa_{x}, \kappa_{y}$, and $\kappa_{z}$ are the components of $\kappa$ in the $\mathrm{x}, \mathrm{y}$, and z directions. $l_{0}$ and $L_{0}$ are the turbulence inner and outer scales. $\varsigma$ is the anisotropic factor and it parameterizes the asymmetry of turbulence cells or eddies in horizontal and vertical directions. When $\varsigma=1$, the isotropic turbulence is shown. As the horizontal turbulence outer cell or eddy is always bigger than the vertical one, $\varsigma$ is always bigger than one.

Based on this spectral model, the theoretical models of the variance of angle of arrival (AOA) fluctuations and the modulation transfer function (MTF) for optical waves propagating through anisotropic non-Kolmogorov turbulence have been derived in $[14,15]$. They are related separately to image distortion and blur, and will be applied in the long range infrared imaging simulation under anisotropic non-Kolmogorov turbulence.

\section{Imaging simulation in anisotropic turbulence}

For the long range infrared imaging simulation in anisotropic non-Kolmogorov turbulence, the major image degradations caused by atmosphere turbulence are image blur and image distortion. The image blur introduced by atmosphere turbulence can be expressed separately with PSF (point spread function) and MTF in spatial and frequency domains. The image distortion can be described by AOA fluctuations of optical wave under atmosphere turbulence. Combining theoretical models of turbulence effects and image processing algorithms, the turbulence-degraded image can be simulated with the following equation:

$$
O(x, y)=R(F(I(x, y)))
$$

$O(x, y)$ and $I(x, y)$ are images with and without turbulence. $F($.$) and R($.$) represent separately$ the operators for image distortion and image blur.

\subsection{Image distortion simulation}

For an input image, image distortion caused by anisotropic non-Kolmogorov turbulence can be simulated with the following steps:

First, calculate the random complex matrix $R_{w}=A+i B$ in frequency domain. $A$ and $B$ are pseudo random number matrixes, which satisfy the Gaussian distribution whose mean value and 
variance equal separately zero and one. $R_{w}$ is introduced to simulate the random fluctuations property of anisotropic non-Kolmogorov turbulence.

Second, calculate the spatial power density function $S_{\beta}(f)$. It describes the spatial distortion relationship between different image regions and is consistent with the real imaging device $[5,6]$ : $S_{\beta}(f)=f^{-b}, b=3$.

Third, filter $S_{\beta}(f)$ with $R_{w}$, and it arrives $H_{\beta} R_{w}=\sqrt{S_{\beta}\left(\sqrt{i^{2}+j^{2}} \Delta \kappa\right)} \Delta \kappa \cdot R_{w}$. With this operation, the constructed random complex filed reflects simultaneously the spatial distortion relationship between different image regions and the random fluctuations property of anisotropic non-Kolmogorov turbulence.

Fourth, fourier inverse transform to $H_{\beta} R_{w}$, and the spatial matrix $\left|\operatorname{IFFT}\left\{H_{\beta} R_{w}\right\}\right|$ will be constructed. The variance of $\left|\operatorname{IFFT}\left\{H_{\beta} R_{w}\right\}\right|$ is set to the variance of AOA fluctuations $\sigma_{A O A}^{2}$ for optical waves propagating through anisotropic non-Kolmogorov turbulence, and the image distortion matrix (with unit of radian) in the horizontal direction is obtained. Repeat these four steps, the image distortion matrix in the vertical direction under anisotropic non-Kolmogorov turbulence is also obtained.

Fifth, divide the image distortion matrixes with angular resolution $\Delta \theta(\Delta \theta=\Delta \delta / L, \Delta \delta$ is the actual size of each pixel and $\mathrm{L}$ is the imaging distance), the image distortion matrixes $\delta x$ and $\delta y$ with the unit of pixels in the horizontal and vertical directions are acquired.

Last, simulate the distorted image under anisotropic non-Kolmogorov turbulence. With $\delta x$ and $\delta y$, and making use of the image bilinear interpolation algorithm, the distorted image under anisotropic non-Kolmogorov turbulence is obtained.

\subsection{Image blur simulation}

To simulate the blurred image under anisotropic non-Kolmogorov turbulence, the isoplanatic angle $\theta_{0}$ need to be calculated. Within this angle, the atmosphere turbulence produces the same blur effects to the image. If exceeds this angle, the image blur at different regions is different. With Rytov approximation theory, $\theta_{0}$ takes the expression as:

$$
\theta_{0}=2.1\left[-\frac{\Gamma(1-\alpha / 2) \cdot 2^{4-\alpha} \cdot \pi^{2} A(\alpha)}{(\alpha-1) \cdot \Gamma(\alpha / 2)}\right]^{1 /(2-\alpha)}\left[\frac{8}{\alpha-2} \cdot \Gamma\left(\frac{2}{\alpha-2}\right)\right]^{\frac{-1}{2}} k^{\frac{-2}{\alpha-2}} \cdot L^{\frac{1-\alpha}{\alpha-2}} \cdot \varsigma\left(\hat{C}_{n}^{2}\right)^{\frac{-1}{\alpha-2}}
$$

In isoplanatic condition, the blur kernel introduced by anisotropic non-Kolmogorov turbulence can be expressed with PSF (can be approximately seen as Gaussian function), which is the inverse Fourier transform of MTF model developed for optical waves propagating through anisotropic non-Kolmogorov turbulence [14]. The blurred image caused by atmosphere turbulence can be simulated by convoluting the original input image with PSF:

$$
I_{\text {blur_iso }}=I \otimes P S F
$$

In non isoplanatic condition, simulation becomes more complex. The main steps are given as follows: First, calculate the random complex matrix $R_{w}=A+i B$ in the frequency domain. This step is the same as that for image distortion simulation. Second, calculate the spatial power density function $S_{\alpha}(f)$. It can reflect the image distortion and coincide with real imaging device [5,6] and takes the form: $S_{\alpha}(f)=f^{-a}, a=3$. This function describes the spatial blur relationship between different image regions. Though $S_{\alpha}(f)$ and $S_{\beta}(f)$ take the same expressions, their physical meanings are different. They represent separately the spatial blur and distortion relationships between different image regions. Third, filter $S_{\alpha}(f)$ with $R_{w}$, and it arrives 
$H_{\alpha} R_{w}=\sqrt{S_{\alpha}\left(\sqrt{i^{2}+j^{2}} \Delta \kappa\right)} \Delta \kappa \cdot R_{w}$. With this operation, the random complex filed which reflects simultaneously the spatial blur relationship between different image regions and the random fluctuations of anisotropic non-Kolmogorov turbulence is constructed. Fourth, fourier inverse transform to $H_{\alpha} R_{w}$, and it obtains the spatial matrix $\left|\operatorname{IFFT}\left\{H_{\alpha} R_{w}\right\}\right|$. The mean value and the mean square error of $\left|\operatorname{IFFT}\left\{H_{\alpha} R_{w}\right\}\right|$ are set separately to $\bar{\sigma}(t)$ and $\sqrt{\bar{\sigma}(t)} \cdot \bar{\sigma}(t)$ is the standard variance of anisotropic turbulence PSF. At this time, the matrix PSF $_{0}$ (with unit of meter) composed by the standard deviation of anisotropic turbulence PSF for all image regions is obtained. The constructed $\mathrm{PSF}_{0}$ considers the anisotropic factor, the general spectral power law, the imaging distance, and the refractive-index structure constant. Dividing $\mathrm{PSF}_{0}$ with $\Delta \delta$, the final matrix $\mathrm{PSF}_{1}$ (with unit of pixels) for all image regions is obtained. Last, convoluting all the input image regions with $\mathrm{PSF}_{1}$, and the blurred image under anisotropic non-Kolmogorov turbulence is obtained.

\section{Simulation results}

In this simulation, the input image is a synthetic image with small target. During the simulation, this input image is repeatedly used to create a simulated image sequence. The parameters in the simulation are set as: $\lambda=1.55 \mu \mathrm{m}, \alpha=10 / 3, \hat{C}_{n}^{2}=1 \times 10^{-14} \mathrm{~m}^{-1 / 3}, L=3 \mathrm{~km}$, the size of image is $512 * 512, \Delta \delta=10 \mathrm{~mm}$, and $D=30 \mathrm{~mm}$.

Figure 1 gives simulation results under different atmosphere turbulence conditions. In order to make comparison with the simulation results under isotropic non-Kolmogorov turbulence, the effective anisotropic factor of one (corresponding to the isotropic turbulence) is also adopted. To well exhibit the small target, the image region around the center (here, the size of $40 * 40$ is adopted) is cropped and shown in Fig. 1 (b)-(g). For these five anisotropic factor values (1, 2, 3, 4, and 5), the theoretical isoplanatic angle $\theta_{0}$ equals $5.25 \mathrm{urad}, 10.55 \mathrm{urad}, 15.83 \mathrm{urad}, 21.1 \mathrm{urad}$, and $26.37 \mathrm{urad}$, respectively. Since the angular resolution for each pixel $\Delta \theta$ is 3.33urad, the size of image region that satisfies local isoplanatic is 2 pixels, 3 pixels, 5pixels, 6 pixels, and 8 pixels for these five anisotropic factor values.

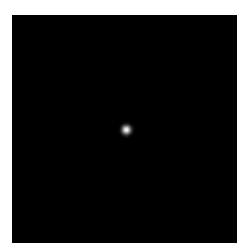

(a)

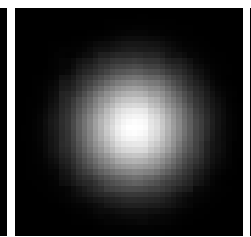

(b)

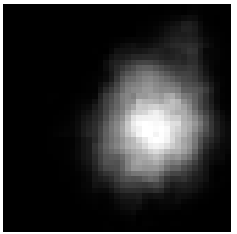

(c)

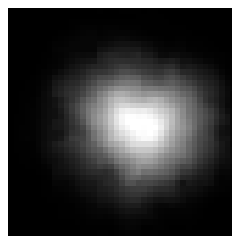

(d)

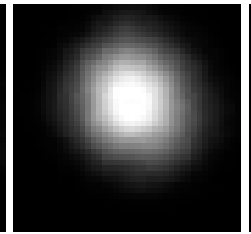

(e)

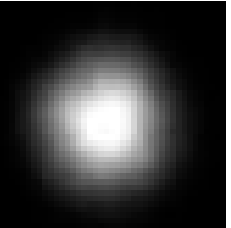

(f)

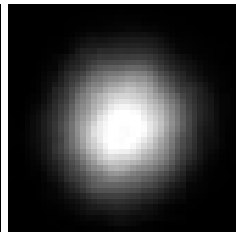

(g)

Fig. 1 Simulation results under different atmosphere turbulence conditions. (a): original input image; (b): the center region (40*40) in original input image; (c-g): simulation results with different anisotropic factor values of 1, 2, 3, 4, and 5.

The centroids of small target under different atmosphere turbulence conditions are calculated and their coordinates in $x$ and $y$ directions are shown in Fig. 2. A sequence of one hundred images are adopted in this analysis. As shown, the centroid of small target fluctuates randomly due to the random fluctuations property of atmosphere turbulence. The mean value and variance of the centroid of small target for the 100 images under different anisotropic turbulence cases are calculated and listed in Table 1. It can be seen that the mean values for the centroid in $\mathrm{x}$ and $\mathrm{y}$ directions are close to 256 which is the centroid coordinate of small target with no turbulence. In addition, the variances for the centroid displacement are different for isotropic and anisotropic turbulence. The centroid of small target fluctuates more severely in isotropic turbulence. With the increase of anisotropic factor, these fluctuations decrease. This phenomenon can be explained physically by the change of curvature of the anisotropic turbulence cells or eddies just as the author stated in [11]. Specifically, these 
anisotropic turbulence cells or eddies act as lenses with a longer radius of curvature compared with the isotropic turbulence ones, and change the focusing properties of the turbulence. When an optical beam propagates along the short axis of anisotropic turbulence cells or eddies, the longer radius of curvature of the anisotropic turbulence cells or eddies make the optical beam less deviated from the direction of propagation. Eventually, the influences of anisotropic turbulence on the optical waves' propagation will be reduced greatly.

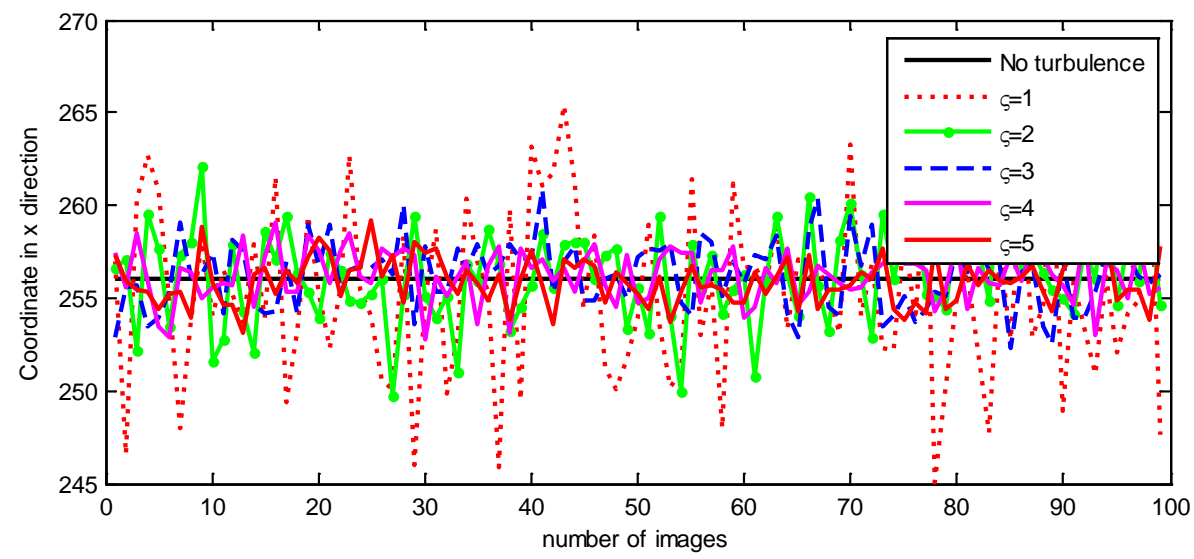

(a)

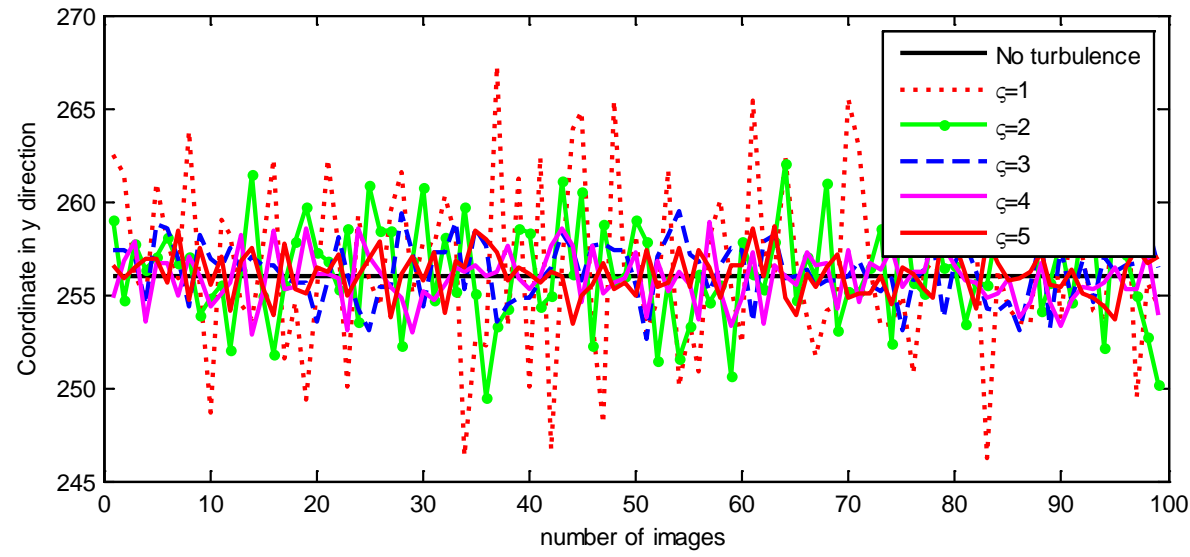

(b)

Fig. 2 Coordinates for the centroid of small target under different atmosphere turbulence conditions. (a) Coordinate in $\mathrm{x}$ direction; (b): Coordinate in y direction.

Table 1. The mean value and variance for the centroid of small target shown Fig. 2.

\begin{tabular}{c|c|c|c|c|c|c}
\hline \multicolumn{2}{c|}{} & $\zeta=1$ & $\zeta=2$ & $\zeta=3$ & $\zeta=4$ & $\zeta=5$ \\
\hline \multirow{2}{*}{ Mean value } & Horizontal & 255.2067 & 256.2425 & 256.2377 & 256.2280 & 255.9304 \\
\cline { 2 - 7 } & Vertical & 256.4758 & 256.3094 & 256.2577 & 255.8513 & 256.1078 \\
\hline \multirow{2}{*}{ Variance } & Horizontal & 20.5103 & 6.3666 & 3.4319 & 1.8890 & 1.6399 \\
\cline { 2 - 7 } & Vertical & 20.7062 & 7.5721 & 2.4262 & 1.8831 & 1.4485 \\
\hline
\end{tabular}

The effective radius for the small target under different atmosphere turbulence conditions is calculated and shown in Fig. 3. If the turbulence does not exist, the effective radius for the small target is about 10 pixels. It can be seen that, the effective radius fluctuates randomly for different images. For the isotorpic turbulence, this kind of fluctuations exhibits the severest. To describe the variation of effective radius for the small target quantitatively, the mean value and variance for the effective radius of small target under different atmosphere turbulence cases are calculated and listed in Table 2. As shown, the istropic turbulence produces more influence on the effective radius of small target than the anisotropic turbulence. Also, when the anisotropic property of atmosphere turbulence exhibits more and more obvious, the variations for the effective radius of small target become smaller and smaller. The physical explanation for this phenomenon is the same as that used to explain Fig. 2. 


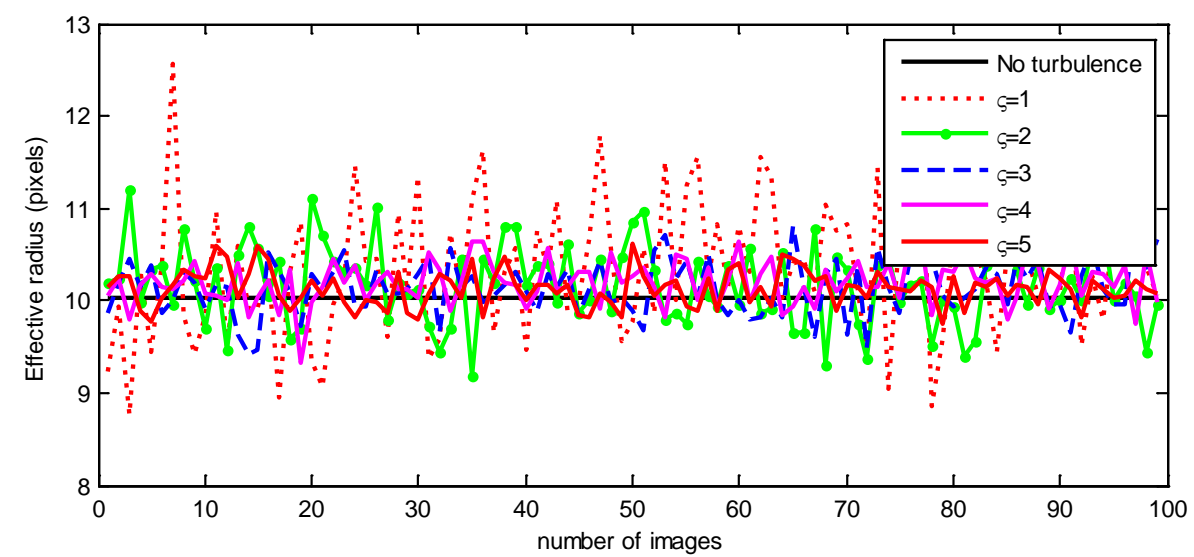

Fig. 3 Effective radius for the small target under different atmosphere turbulence conditions

Table 2. The mean and variance for the effective radius of small target shown in Fig. 3

\begin{tabular}{c|c|c|c|c|c}
\hline & $\zeta=1$ & $\zeta=2$ & $\zeta=3$ & $\zeta=4$ & $\zeta=5$ \\
\hline Mean value & 10.3662 & 10.1776 & 10.1429 & 10.1992 & 10.1339 \\
\hline Variance & 0.5786 & 0.1821 & 0.0903 & 0.0596 & 0.0383 \\
\hline
\end{tabular}

\section{Conclusions and discussions}

In this study, the infrared imaging through anisotropic non-Kolmogorov turbulence is simulated. The theoretical turbulence effects models of optical wave under anisotropic non-Kolmogorov turbulence are adopted, which consider simultaneously the effective anisotropic factor which parameterizes the asymmetry of turbulence cells or eddies in the horizontal and vertical directions, the general spectral power law, the imaging distance, the wavelength, and the receiver aperture. Based on this, the imaging processing algorithms, composing of image convolution and image bilinear interpolation, are applied in the simulation. Results show that with the increase of anisotropic factor, the anisotropic non-Kolmogorov turbulence produces less effect on the image displacement and image blur. Due to the lack of sufficient experiments about the anisotropic turbulence, simulations performed in this investigation cannot be compared with the real obtained images. Despite of this, the researches done in this study help better investigate the long range infrared imaging through anisotropic turbulence.

\section{References}

[1] N. Roddier N, “Atmospheric wavefront simulation using Zernike polynomials,” Opt. Eng., 29(10): 1174-1180 (1990).

[2] A. Beghi A, A. Cenedese, and A. Masiero A, "A Markov-Random-Field-based approach to modeling and prediction of atmospheric turbulence," IEEE 16th Mediterranean Conference on Control and Automation, 1735-1740 (2008).

[3] B. Zhang, S. Qin, and X. Wang, "Accurate and fast simulation of Kolmogorov phase screen by combing spectral method with Zernike polynomials method,” Chin.Opt.Lett., 8(10): 969-971, (2010).

[4] V. S. R. Gudimetla, R. B. Holmes, T. C. Farrell, and J. Lucas, "Phase screen simulations of laser propagation through non-Kolmogorov atmospheric turbulence,” Proc.SPIE, 8038: 803808-1-12, (2011).

[5] E. Repasi and R.Weiss, "Computer Simulation of Image Degradations by Atmospheric Turbulence for Horizontal Views,” Proc. SPIE, 8014: 80140U-1-9 (2011).

[6] D. Oxford and R. L. Espinola, "Simulation of a laser range-gated SWIR imaging system in weak turbulence conditions ,” Proc. SPIE, 8014: 80140T-1-12 (2011).

[7] K. R. Leonard and R. L. Espinola, "Validation of atmospheric turbulence simulations of extended scenes,” Proc. SPIE, 9071: 907118 (2014). 
[8] T. D. Bosq and E. Repasi, "Detector Integration Time Dependent Atmospheric Turbulence Imaging Simulation,” Proc. SPIE, 9452: 94520B (2015).

[9] M. S. Belen'kii, E. Cuellar, K. A. Hughes, and V. A. Rye, "Experimental study of spatial structure of turbulence at Maui Space Surveillance Site (MSSS),” Proc. SPIE, 6304: 63040U (2006).

[10] C. Robert, J. M. Conan, V. Michau, J. B. Renard, C. Robert, and F. Dalaudier, "Retrieving parameters of the anisotropic refractive index fluctuations spectrum in the stratosphere from balloon-borne observations of stellar scintillation,” J. Opt. Soc. Am. A, 25(2): 379-393 (2008).

[11] I. Toselli, B. Agrawal, and S. Restaino, "Light propagation through anisotropic turbulence,” J. Opt. Soc. Am. A, 28(3): 483-488 (2011).

[12] V. S. Rao Gudimetla, R. B. Holmes, and J. F. Riker, "Analytical expressions for the log-amplitude correlation function for plane wave propagation in anisotropic non-Kolmogorov refractive turbulence,” J. Opt. Soc. Am. A, 29(12): 2622-2627 (2012).

[13] I. Toselli, "Introducing the concept of anisotropy at different scales for modeling optical turbulence,” J. Opt. Soc. Am. A, 31(8):1868-1875 (2014).

[14] L. Y. Cui, B. D. Xue, X. G. Cao, and F. G. Zhou, "Atmospheric turbulence MTF for optical waves' propagation through anisotropic non-Kolmogorov atmospheric turbulence,” Optics \& Laser Technology, 63: 70-75 (2014).

[15] L. Y. Cui, “Analysis of angle of arrival fluctuations for optical waves' propagation through weak anisotropic non-Kolmogorov turbulence,” Opt. Express, 23(5): 6313-6325 (2015). 